\title{
DECOMPOSITIONS OF COUNTABLE LINEAR TRANSFORMATIONS
}

\author{
HUANYIN CHEN \\ Department of Mathematics, Hangzhou Normal University \\ Hangzhou 310036, China \\ e-mail: huanyinchen@yahoo.cn \\ http://huanyinchens.blogbus.com
}

(Received 2 February 2009; accepted 10 October 2009; first published online 22 March 2010)

\begin{abstract}
Let $V$ be a countably generated right vector space over a division ring $D$. If $D ¥ \mathbb{Z} / 2 \mathbb{Z}, \mathbb{Z} / 3 \mathbb{Z}$, then for any $\gamma \in \operatorname{End}_{D}(V)$, there exists $\alpha \in A u t_{D}(V)$ such that $\gamma+\alpha, \gamma-\alpha^{-1} \in A u t_{D}(V)$. This gives a generalization of [D. Zelinsky, Proc. Amer. Math. Soc. 5 (1954), 627-630, Theorem].
\end{abstract}

\section{Mathematics Subject Classification.}

In [6, Theorem], Zelinsky proved that every linear transformation over a division ring is the sum of two automorphisms with the exception of the identity transformation on a space of two elements. In [5], Nicholson and Varadarjan proved that every linear transformation over an infinite-dimensional vector space is the sum of an idempotent and an automorphism. The main purpose of this paper is to generalize Zelinsky's result and investigate new decompositions of countably linear transformations. Let $V$ be a countably generated right vector space over a division $\operatorname{ring} D$. If $D \neq \mathbb{Z} / 2 \mathbb{Z}, \mathbb{Z} / 3 \mathbb{Z}$, we prove that for any $\gamma \in \operatorname{End}_{D}(V)$, there exists $\alpha \in A u t_{D}(V)$ such that $\gamma+\alpha, \gamma-\alpha^{-1} \in$ $A u t_{D}(V)$. Furthermore, there exists $\sigma \in A u t_{D}(V)$ such that $\gamma^{2}+\sigma \gamma+1 \in \operatorname{Aut}_{D}(V)$.

Throughout, $G L_{n}(D)$ denotes the $n$-dimensional general linear group over a division ring $D$ and $M_{n}(D)$ denote the ring of all $n \times n$ matrices over $D$ with an identity $I_{n}$. We use $D^{*}$ to denote the set of all non-zero elements in $D$ and $\mathbb{N}$ to denote the set of all natural numbers. Let $V$ be a countably generated right vector space over a division ring $D . \operatorname{End}_{D}(V)$ and $A u t_{D}(V)$ stand for the sets of all endomorphisms and all automorphisms on $V$, respectively. Let $\left\{x_{1}, x_{2}, \cdots, x_{n}, \cdots\right\}$ be a basis of $V$. We say that $\sigma: V \rightarrow V$ is a shift operator if $\sigma\left(x_{k}\right)=x_{k+1}$ for all $k \in \mathbb{N}$.

LEMMA 1. Let $V$ be a countably generated right vector space over a division ring $D$ and $\sigma \in \operatorname{End}_{D}(V)$ be a shift operator. Then there exists $\alpha \in \operatorname{Aut}_{D}(V)$ such that $\sigma+$ $\alpha, \sigma-\alpha^{-1} \in \operatorname{Aut}_{D}(V)$.

Proof. Let

$$
C=\left(\begin{array}{ccc}
0 & 0 & 0 \\
1_{D} & 0 & 0 \\
0 & 1_{D} & 0
\end{array}\right), \quad L=\left(\begin{array}{ccc}
0 & 0 & 1_{D} \\
0 & 0 & 0 \\
0 & 0 & 0
\end{array}\right)
$$


and let

$$
A=\left(\begin{array}{cccc}
C & 0 & 0 & \cdots \\
L & C & 0 & \cdots \\
0 & L & C & \cdots \\
\vdots & \vdots & \vdots & \ddots
\end{array}\right)
$$

Then $A$ is a column-finite matrix over $D$. It is easy to verify that $C^{3}=L^{2}=0$. Let

$$
B=\left(\begin{array}{cccc}
C & 0 & 0 & \ldots \\
0 & C & 0 & \ldots \\
0 & 0 & C & \ldots \\
\vdots & \vdots & \vdots & \ddots
\end{array}\right), D=\left(\begin{array}{cccc}
0 & 0 & 0 & \cdots \\
L & 0 & 0 & \ldots \\
0 & L & 0 & \ldots \\
\vdots & \vdots & \vdots & \ddots
\end{array}\right)
$$

Then

$$
A=\left(\begin{array}{cccc}
C & 0 & 0 & \cdots \\
L & C & 0 & \ldots \\
0 & L & C & \ldots \\
\vdots & \vdots & \vdots & \ddots
\end{array}\right)=B+D
$$

Choose a basis $\left\{x_{1}, x_{2}, x_{3}, \cdots\right\}$ of $V$. Construct two transformations $\varepsilon, \delta \in \operatorname{End}_{D}(V)$ given by

$$
\begin{aligned}
& \varepsilon\left(x_{1}, x_{2}, x_{3}, \ldots\right)=\left(x_{1}, x_{2}, x_{3}, \ldots\right) B \\
& \delta\left(x_{1}, x_{2}, x_{3}, \ldots\right)=\left(x_{1}, x_{2}, x_{3}, \ldots\right) D
\end{aligned}
$$

Then

$$
\begin{aligned}
(\varepsilon & +\delta)\left(x_{1}, x_{2}, x_{3}, \ldots\right) \\
& =\left(x_{1}, x_{2}, x_{3}, \ldots\right)(B+D) \\
& =\left(x_{1}, x_{2}, x_{3}, \ldots\right)\left(\begin{array}{cccc}
C & 0 & 0 & \ldots \\
L & C & 0 & \ldots \\
0 & L & C & \ldots \\
\vdots & \vdots & \vdots & \ddots
\end{array}\right) \\
& =\sigma\left(x_{1}, x_{2}, x_{3}, \ldots\right),
\end{aligned}
$$

and then $\sigma=\varepsilon+\delta$. Clearly, $\varepsilon^{3}=\delta^{2}=0$. Thus, $\sigma=(\delta+1)+(\varepsilon-1)$ and $\sigma=(\delta-$ $1)+(\varepsilon+1)$. Let $\alpha=-(\delta+1)$. Then $\alpha^{-1}=\delta-1$. It is easy to verify that $(\varepsilon-1)^{-1}=$ $-\varepsilon^{2}-\varepsilon-1$ and $(\varepsilon+1)^{-1}=\varepsilon^{2}-\varepsilon+1$. Therefore, $\sigma+\alpha, \sigma-\alpha^{-1} \in A t_{D}(V)$, as asserted.

LeMmA 2. Let $D$ be a division ring and $n \in \mathbb{N}$. If $D \neq \mathbb{Z} / 2 \mathbb{Z}, \mathbb{Z} / 3 \mathbb{Z}$, then for any $\gamma \in M_{n}(D)$, there exists $\alpha \in G L_{n}(D)$ such that $\gamma+\alpha, \gamma-\alpha^{-1} \in G L_{n}(D)$.

Proof. Assume that $D \neq \mathbb{Z} / 2 \mathbb{Z}, \mathbb{Z} / 3 \mathbb{Z}$. Then $|D| \geq 4$. Let $x, y \in D$. If $x=y=0$, then $x+u, y-u^{-1} \in D^{*}$ with $u=1_{D}$. If $x \neq 0$, then we choose $v \in D$ such that 
$v \notin\left\{0, y,-x^{-1}\right\}$. Set $u=v^{-1}$. Then, $x+u, y-u^{-1} \in D^{*}$. If $y \neq 0$, then we choose $u \in D$ such that $u \notin\left\{0,-x, y^{-1}\right\}$. Hence, $x+u, y-u^{-1} \in D^{*}$. Therefore, for any $x, y \in D$, we can find $u \in D^{*}$ such that $x+u, y-u^{-1} \in D^{*}$.

Given any $\beta, \gamma \in M_{n}(D)$, we can find $\beta_{11}, \gamma_{11} \in D, \beta_{12}, \gamma_{12} \in M_{1 \times(n-1)}(D)$, $\beta_{21}, \gamma_{21} \in M_{(n-1) \times 1}(D)$ and $\beta_{22}, \gamma_{22} \in M_{(n-1) \times(n-1)}(D)$ such that

$$
\beta=\left(\begin{array}{ll}
\beta_{11} & \beta_{12} \\
\beta_{21} & \beta_{22}
\end{array}\right), \quad \gamma=\left(\begin{array}{ll}
\gamma_{11} & \gamma_{12} \\
\gamma_{21} & \gamma_{22}
\end{array}\right) .
$$

It will suffice to prove that $\beta+\delta, \gamma-\delta^{-1} \in G L_{n}(D)$ for some $\delta \in G L_{n}(D)$. From the previous discussion, we can find $a_{1}, a_{2} \in D^{*}$ such that $\beta_{11}+a=u_{1} \in D^{*}$ and $\gamma_{11}-$ $a^{-1}=u_{2} \in D^{*}$. Since $\beta_{22}-\beta_{21} u_{1}^{-1} \beta_{12}, \gamma_{22}-\gamma_{21} u_{2}^{-1} \gamma_{12} \in M_{n-1}(D)$, from induction hypothesis, we can find $b \in G L_{n-1}(D)$ such that $\beta_{22}-\beta_{21} u_{1}^{-1} \beta_{12}+b=v_{1} \in G L_{n-1}(D)$ and $\gamma_{22}-\gamma_{21} u_{2}^{-1} \gamma_{12}-b^{-1}=v_{2} \in G L_{n-1}(D)$. As a result, we deduce that

$$
\begin{aligned}
& \beta+\operatorname{diag}(a, b)=\left(\begin{array}{cc}
u_{1} & \beta_{12} \\
\beta_{21} & v_{1}+\beta_{21} u_{1}^{-1} \beta_{12}
\end{array}\right), \\
& \gamma-\operatorname{diag}\left(a^{-1}, b^{-1}\right)=\left(\begin{array}{cc}
u_{2} & \gamma_{12} \\
\gamma_{21} & v_{2}+\gamma_{21} u_{2}^{-1} \gamma_{12}
\end{array}\right)
\end{aligned}
$$

Clearly,

$$
\begin{aligned}
\left(\begin{array}{cc}
u_{1} & \beta_{12} \\
\beta_{21} & v_{1}+\beta_{21} u_{1}^{-1} \beta_{12}
\end{array}\right) & =\left(\begin{array}{cc}
1 & 0 \\
\beta_{21} u_{1}^{-1} & 1
\end{array}\right)\left(\begin{array}{cc}
u_{1} & \beta_{12} \\
0 & v_{1}
\end{array}\right), \\
\left(\begin{array}{cc}
u_{2} & \gamma_{12} \\
\gamma_{21} & v_{2}+\gamma_{21} u_{2}^{-1} \gamma_{12}
\end{array}\right) & =\left(\begin{array}{cc}
1 & 0 \\
\gamma_{21} u_{2}^{-1} & 1
\end{array}\right)\left(\begin{array}{cc}
u_{2} & \gamma_{12} \\
0 & v_{2}
\end{array}\right) .
\end{aligned}
$$

Thus,

$$
\left(\begin{array}{cc}
u_{1} & \beta_{12} \\
\beta_{21} & v_{1}+\beta_{21} u_{1}^{-1} \beta_{12}
\end{array}\right), \quad\left(\begin{array}{cc}
u_{2} & \gamma_{12} \\
\gamma_{21} & v_{2}+\gamma_{21} u_{2}^{-1} \gamma_{12}
\end{array}\right) \in G L_{n-1}(D)
$$

By induction, we conclude that $\beta+\delta, \gamma-\delta^{-1} \in G L_{n}(D)$ for some $\delta \in G L_{n}(D)$. Choose $\beta=\gamma$. Then we obtain the result.

Let $D=\mathbb{Z} / 3 \mathbb{Z}$. Choose $x=\overline{2} \in D$. We see that there is no $u \in D^{*}$ such that $x+$ $u, x-u^{-1} \in D^{*}$. But we see that for any $x \in D$, there exists a $u \in D^{*}$ such that $x-$ $u, x-u^{-1} \in D^{*}$. Thus, the later condition is far from the previous.

LEMMA 3. Let $V$ be a countably generated right vector space over a division ring $D$, and let $\gamma \in \operatorname{End}_{D}(V)$ be such that $V$ is spanned by $\left\{y, \gamma(y), \gamma^{2}(y), \ldots\right\}$. If $D \neq$ $\mathbb{Z} / 2 \mathbb{Z}, \mathbb{Z} / 3 \mathbb{Z}$, then there exists $\alpha \in A u t_{D}(V)$ such that $\gamma+\alpha, \gamma-\alpha^{-1} \in \operatorname{Aut}_{D}(V)$.

Proof. Let $V \neq 0$. If $\gamma^{n}(y) \notin y D+\gamma(y) D+\gamma^{2}(y) D+\cdots+\gamma^{n-1}(y) D$ for all $n \in \mathbb{N}$, then $\left\{y, \gamma(y), \gamma^{2}(y), \ldots\right\}$ forms a basis of $V$. Since $\gamma$ is a shift operator with respect to this basis, by virtue of Lemma 1 , we have $\alpha \in A u t_{D}(V)$ such that $\gamma+\alpha, \gamma-\alpha^{-1} \in$ $\mathrm{Aut}_{D}(V)$. 
Suppose there exists some $n \in \mathbb{N}$ such that $\gamma^{n}(y) \in y D+\gamma(y) D+\gamma^{2}(y) D+\cdots+$ $\gamma^{n-1}(y) D$. If $n$ is minimal with this property, then $\left\{y, \gamma(y), \gamma^{2}(y), \ldots, \gamma^{n-1}(y)\right\}$ forms a basis of $V$; hence, $\operatorname{End}_{D}(V) \cong M_{n}(D)$. By virtue of Lemma 2, the result follows.

LeMma 4. Let $V$ be a countably generated right vector space over a division ring $D, D \neq \mathbb{Z} / 2 \mathbb{Z}, \mathbb{Z} / 3 \mathbb{Z}$, let $\gamma \in \operatorname{End}_{D}(V)$ and $U$ be a $\gamma$-invariant vector subspace of $V$. Assume that $V=U+K$ where $K=y D+\gamma(y) D+\gamma^{2}(y) D+\cdots$ for some $y \in V$. If there exists $\alpha^{\prime} \in \operatorname{Aut}_{D}(U)$ such that $\left.\gamma\right|_{U}+\alpha^{\prime},\left.\gamma\right|_{U}-\left(\alpha^{\prime}\right)^{-1} \in A^{\prime} t_{D}(U)$, then there exists $\alpha \in \operatorname{Aut}_{D}(V)$ such that $\gamma+\alpha, \gamma-\alpha^{-1} \in \operatorname{Aut}_{D}(V)$.

Proof. Obviously, we have a vector subspace $M$ such that $V=M \oplus U$. Since $U$ is $\gamma$-invariant, we have a transformation $\bar{\gamma}: V / U \rightarrow V / U$ defined by $\bar{\gamma}(\bar{v})=\overline{\gamma(v)}$. Let $\theta: V \rightarrow V$ given by $\theta(m+u)=m$ for any $m \in M, u \in U$. Then $\theta(V)=M$ and $\theta^{2}=\theta$. This induces a $D$-isomorphism $\theta_{0}: V / U \rightarrow M$. Let $\zeta=\theta_{0} \bar{\gamma} \theta_{0}^{-1} \in \operatorname{End}_{D}(M)$. Then $\zeta \theta_{0}=\theta_{0} \bar{\gamma}$, and so $\zeta \theta_{0}(\bar{v})=\theta_{0} \bar{\gamma}(\bar{v})$ for any $v \in V$. This induces that $\zeta \theta(v)=\theta \gamma(v)$. For any $m \in M, \zeta(m)=\zeta \theta(m)=\theta \gamma(m)$. Hence, $\theta \zeta(m)=\theta^{2} \gamma(m)=\theta \gamma(m)$. It follows that $\gamma(m)-\zeta(m) \in \operatorname{Ker} \theta=U$ for all $m \in M$.

By hypothesis, we see that $\left\{\bar{y}, \bar{\gamma}(\bar{y}),(\bar{\gamma})^{2}(\bar{y}), \ldots\right\}$ spans $V / U$, and then $\left\{\theta_{0}(\bar{y}), \theta_{0}(\bar{\gamma}(\bar{y})), \theta_{0}\left((\bar{\gamma})^{2}(\bar{y})\right), \ldots\right\}$ spans $M$. Clearly, we have $\theta_{0}\left((\bar{\gamma})^{n}(\bar{y})\right)=\zeta^{n}\left(\theta_{0}(\bar{y})\right)$ for all $n \in \mathbb{N}$, and so $\left\{\theta_{0}(\bar{y}), \zeta\left(\theta_{0}(\bar{y})\right), \zeta^{2}\left(\theta_{0}(\bar{y})\right), \ldots\right\}$ spans $M$. In light of Lemma 3, there exists $\alpha_{1} \in \operatorname{Aut}_{D}(M)$ such that $\zeta+\alpha_{1}, \zeta-\left(\alpha_{1}\right)^{-1} \in \operatorname{Aut}_{D}(M)$. By assumption, there exists $\alpha^{\prime} \in A u t_{D}(U)$ such that $\left.\gamma\right|_{U}+\alpha^{\prime},\left.\gamma\right|_{U}-\left(\alpha^{\prime}\right)^{-1} \in A u t_{D}(U)$. Define $\alpha: V \rightarrow V$ given by $\alpha(m+u)=\alpha_{1}(m)+\alpha^{\prime}(u)$ for any $m \in M, u \in U$. Let $\beta_{1}=\zeta+\alpha_{1}$ and $\beta^{\prime}=$ $\left.\gamma\right|_{U}+\alpha^{\prime}$. Let $\beta: V \rightarrow V$ given by $\beta(m+u)=\beta_{1}(m)+\left(\beta^{\prime}(u)+\gamma(m)-\zeta(m)\right)$ for any $m \in M, u \in U$. One easily checks that $\alpha \in \operatorname{Aut}_{D}(V)$. Furthermore, we see that

$$
\begin{aligned}
& (-\alpha+\beta)(m+u) \\
& \quad=-\alpha_{1}(m)-\alpha^{\prime}(u)+\beta_{1}(m)+\left(\beta^{\prime}(u)+\gamma(m)-\zeta(m)\right) \\
& \quad=\left(-\alpha_{1}(m)+\beta_{1}(m)\right)+\left(-\alpha^{\prime}(u)+\beta^{\prime}(u)\right)+\gamma(m)-\zeta(m) \\
& \quad=\gamma(u)+\gamma(m) \\
& \quad=\gamma(m+u)
\end{aligned}
$$

for any $m \in M, u \in U$. Thus, $\gamma+\alpha=\beta$. If $\beta(m+u)=0$, then $\beta_{1}(m)=-\beta^{\prime}(u)-$ $\gamma(m)+\zeta(m) \in M \cap U=0$; hence, $\beta_{1}(m)=0$. This infers that $m=0$. Furthermore, we get $-\beta^{\prime}(u)=0$, and so $u=0$. Thus $\beta$ is a $D$-monomorphism. Obviously, $U \subseteq \operatorname{Im} \beta$. It suffices to show that $M \subseteq \operatorname{Im} \beta$. If $m \in M$, we can find $m_{0} \in M$ such that $m=\beta_{1}\left(m_{0}\right)$. Also we have $u_{0} \in U$ such that $\gamma\left(m_{0}\right)-\zeta\left(m_{0}\right)=\beta^{\prime}\left(u_{0}\right)$. It is easy to check that

$$
\begin{aligned}
& \beta\left(m_{0}+u_{0}\right) \\
& \quad=\beta_{1}\left(m_{0}\right)+\left(\beta^{\prime}\left(u_{0}\right)+\gamma\left(m_{0}\right)-\zeta\left(m_{0}\right)\right) \\
& \quad=m .
\end{aligned}
$$

That is, $\beta$ is a $D$-epimorphism, and so $\gamma+\alpha=\beta \in A u t_{D}(V)$.

Let $\sigma_{1}=\zeta-\left(\alpha_{1}\right)^{-1}$ and $\sigma^{\prime}=\left.\gamma\right|_{U}-\left(\alpha^{\prime}\right)^{-1}$. Let $\delta: V \rightarrow V$ given by $\delta(m+u)=$ $\sigma_{1}(m)+\left(\sigma^{\prime}(u)+\gamma(m)-\zeta(m)\right)$ for any $m \in M, u \in U$. Then

$$
\begin{aligned}
& \left(\alpha^{-1}+\delta\right)(m+u) \\
& \quad=\alpha_{1}^{-1}(m)+\left(\alpha^{\prime}\right)^{-1}(u)+\sigma_{1}(m)+\left(\sigma^{\prime}(u)+\gamma(m)-\zeta(m)\right) \\
& \quad=\left(\alpha_{1}^{-1}(m)+\sigma_{1}(m)\right)+\left(\left(\alpha^{\prime}\right)^{-1}(u)+\sigma^{\prime}(u)\right)+\gamma(m)-\zeta(m) \\
& \quad=\gamma(u)+\gamma(m) \\
& \quad=\gamma(m+u)
\end{aligned}
$$


for any $m \in M, u \in U$. So, $\gamma-\alpha^{-1}=\delta$. Analogously, we see that $\delta \in \operatorname{Aut}_{D}(V)$. Therefore, we complete the proof.

THEOREM 5. Let $V$ be a countably generated right vector space over a division ring $D$, and let $\gamma \in \operatorname{End}_{D}(V)$. If $D ¥ \mathbb{Z} / 2 \mathbb{Z}, \mathbb{Z} / 3 \mathbb{Z}$, then there exists $\alpha \in A u t_{D}(V)$ such that $\gamma+\alpha, \gamma-\alpha^{-1} \in A u t_{D}(V)$.

Proof. Let $\gamma \in \operatorname{End}_{D}(V)$ and $\Omega=\left\{(U, \sigma, \pi) \mid U_{D} \subseteq V\right.$ is $\gamma$-invariant, $\left.\gamma\right|_{U}+\sigma=$ $\left.\pi,\left.\gamma\right|_{U}-\sigma^{-1}=\tau, \sigma, \pi, \tau \in A u t_{D}(U)\right\}$. Partially order $\Omega$ by writing $(U, \sigma, \pi) \leq$ $\left(U^{\prime}, \sigma^{\prime}, \pi^{\prime}\right)$ if $U \subseteq U^{\prime}, \sigma=\left.\sigma^{\prime}\right|_{U}, \pi=\left.\pi^{\prime}\right|_{U}$. Given $\left(U_{1}, \sigma_{1}, \pi_{1}\right) \leq\left(U_{2}, \sigma_{2}, \pi_{2}\right) \leq \cdots$ in $\Omega$, then $\bigcup_{i=1}^{\infty} U_{i}$ is a $\gamma$-invariant vector subspace of $V$. Define $\sigma: \bigcup_{i=1}^{\infty} U_{i} \rightarrow \bigcup_{i=1}^{\infty} U_{i}$ given by $\sigma\left(x_{i}\right)=\sigma_{i}\left(x_{i}\right)$ for any $x_{i} \in U_{i}$ and $\pi: \bigcup_{i=1}^{\infty} U_{i} \rightarrow \bigcup_{i=1}^{\infty} U_{i}$ given by $\pi\left(x_{i}\right)=$ $\pi_{i}\left(x_{i}\right)$ for any $x_{i} \in U_{i}$. Then $\left.\gamma\right|_{\bigcup_{i=1}^{\infty} U_{i}}+\sigma,\left.\gamma\right|_{\bigcup_{i=1}^{\infty} U_{i}}-\sigma^{-1} \in A u t_{D}\left(\bigcup_{i=1}^{\infty} U_{i}\right)$. As a result, $\left(\bigcup_{i=1}^{\infty} U_{i}, \sigma, \pi\right) \in \Omega$, i.e. $\Omega$ is inductive. It follows from Zorn's lemma that there exists $(W, \alpha, \beta) \in \Omega$ which is maximal in $\Omega$. If $W \neq V$, then we can find a $y \in V-W$. Choose $K=y D+\gamma(y) D+\gamma^{2}(y) D+\cdots$ and $W_{0}=W+K$. Then $W_{0}$ is $\gamma$-invariant. In light of Lemma $4, W_{0} \in \Omega$. This gives a contradiction. Therefore, we conclude that $V=W$, as required.

If $R$ is a strongly $\pi$-regular ring, then for any $x \in R$ there exists an idempotent $e \in R$ such that $x^{2}+e x+1 \in U(R)$ (see [1, Corollary 13]). For countable linear transformations over vector spaces, we can derive the following.

COROLlary 6. Let $V$ be a countably generated right vector space over a division ring $D$, and let $\gamma \in \operatorname{End}_{D}(V)$. If $D ¥ \mathbb{Z} / 2 \mathbb{Z}, \mathbb{Z} / 3 \mathbb{Z}$, then there exists $\alpha \in \operatorname{Aut}_{D}(V)$ such that $\gamma^{2}+\alpha \gamma+1 \in \operatorname{Aut}_{D}(v)$.

Proof. In view of Theorem 5, there exists some $\varepsilon \in A u t_{D}(V)$ such that $-\gamma+$ $\varepsilon,-\gamma-\varepsilon^{-1} \in A u t_{D}(V)$. Let $-\gamma+\varepsilon=\alpha$ and $-\gamma-\varepsilon^{-1}=\sigma$. Then $\varepsilon \gamma+1=-\varepsilon \sigma$, this yields that $(\gamma+\alpha) \gamma+1=-\varepsilon \sigma$. Therefore, $\gamma^{2}+\alpha \gamma+1 \in A u t_{D}(V)$, as asserted.

EXAMPLE 7. Let $V=\mathbb{Z} / 3 \mathbb{Z}$ be the one-dimensional vector space over $\mathbb{Z} / 3 \mathbb{Z}$. Then for any $\gamma \in \operatorname{End}_{D}(V)$, there exists $\alpha \in A u t_{D}(V)$ such that $\gamma^{2}+\alpha \gamma+1 \in \operatorname{Aut}_{D}(V)$. But for some $\gamma \in \operatorname{End}_{D}(V)$, there is no any $\alpha \in \operatorname{Aut}_{D}(V)$ such that $\gamma+\alpha, \gamma-\alpha^{-1} \in \operatorname{Aut}_{D}(V)$.

Proof. Straightforward.

We note that the conditions $D \neq \mathbb{Z} / 2 \mathbb{Z}, \mathbb{Z} / 3 \mathbb{Z}$ in Theorem 5 are not necessary as the following shows.

EXAMPLE 8. Let $V=\mathbb{Z} / 2 \mathbb{Z} \oplus \mathbb{Z} / 2 \mathbb{Z}$ be the two-dimensional vector space over $\mathbb{Z} / 2 \mathbb{Z}$, and let $\gamma \in \operatorname{End}_{\mathbb{Z} / 2 \mathbb{Z}}(V)$. Then there exists $\alpha \in \operatorname{Aut}_{\mathbb{Z} / 2 \mathbb{Z}}(V)$ such that $\gamma+\alpha, \gamma-\alpha^{-1} \in$ $\operatorname{Aut}_{\mathbb{Z} / 2 \mathbb{Z}}(V)$, and so $\gamma^{2}+\alpha \gamma+1 \in \operatorname{Aut}_{\mathbb{Z} / 2 \mathbb{Z}}(V)$.

Proof. Clearly, we see that

$$
G L_{2}(\mathbb{Z} / 2 \mathbb{Z})=\left\{\left(\begin{array}{ll}
0 & 1 \\
1 & 0
\end{array}\right),\left(\begin{array}{ll}
0 & 1 \\
1 & 1
\end{array}\right),\left(\begin{array}{ll}
1 & 0 \\
0 & 1
\end{array}\right),\left(\begin{array}{ll}
1 & 0 \\
1 & 1
\end{array}\right),\left(\begin{array}{ll}
1 & 1 \\
0 & 1
\end{array}\right),\left(\begin{array}{ll}
1 & 1 \\
1 & 0
\end{array}\right)\right\} .
$$

For any $\gamma \in M_{2}(\mathbb{Z} / 2 \mathbb{Z})$. One directly checks that there exists $\alpha \in G L_{2}(\mathbb{Z} / 2 \mathbb{Z})$ such that $\gamma+\alpha, \gamma-\alpha^{-1} \in G L_{2}(\mathbb{Z} / 2 \mathbb{Z})$, as required. 
Let $R=E n d_{\mathbb{Z} / 2 \mathbb{Z}}(\mathbb{Z} / 2 \mathbb{Z} \oplus \mathbb{Z} / 2 \mathbb{Z})$. Example 8 means that for any $\gamma \in R$, there exists $\alpha \in U(R)$ such that $\gamma+\alpha, \gamma-\alpha^{-1} \in U(R)$. A natural problem is to ask whether such condition for a ring is Morita invariant. The answer is negative. It follows from Example 8 that $M_{2}(\mathbb{Z} / 2 \mathbb{Z})$ satisfies such condition, while $\mathbb{Z} / 2 \mathbb{Z}$ does not satisfy this one.

Recall that a ring $R$ satisfies unit 1-stable range provided that $a R+b R=R$ with $a, b \in R$ implies that there exists $u \in U(R)$ such that $a+b u \in U(R)$. A ring $R$ satisfies Goodearl-Menal condition provided that for any $x, y \in R$, there exists $u \in U(R)$ such that $x-u, y-u^{-1} \in U(R)$ (cf. [2]). As it is well known, $M_{n}(\mathbb{Z} / 2 \mathbb{Z})(n \geq 3)$ and $M_{n}(\mathbb{Z} / 3 \mathbb{Z})(n \geq 2)$ satisfy the Goodearl-Menal condition. From these, we can derive the following.

Proposition 9. Let $R$ be a semi-local ring. Then the following are equivalent:

(1) For any $\gamma \in R$, there exists $\alpha \in U(R)$ such that $\gamma+\alpha, \gamma-\alpha^{-1} \in U(R)$.

(2) $R$ has no homomorphic image $\mathbb{Z} / 2 \mathbb{Z}, \mathbb{Z} / 3 \mathbb{Z}$.

Proof. (1) $\Rightarrow$ (2) If $R$ has $\mathbb{Z} / 2 \mathbb{Z}$ as a homomorphic image, then there exists an ideal $I$ of $R$ such that $R / I \cong \mathbb{Z} / 2 \mathbb{Z}$. By hypothesis, there exists some $u \in U(R)$ such that $\overline{1}-\bar{u} \in U(R / I)$. Hence, $\overline{1}-\overline{1}=\overline{1}$ in $\mathbb{Z} / 2 \mathbb{Z}$, a contradiction. Thus $R$ does not have $\mathbb{Z} / 2 \mathbb{Z}$ as a homomorphic image. Likewise, it follows from Example 7 that $R$ does not have $\mathbb{Z} / 3 \mathbb{Z}$ as a homomorphic image.

(2) $\Rightarrow$ (1) Since $R$ is semi-local, there exist division rings $D_{1}, \ldots, D_{m}$ such that $R / J(R) \cong \bigoplus_{i=1}^{m} M_{n_{i}}\left(D_{i}\right)$. By hypothesis, either $D_{i} \supsetneqq \mathbb{Z} / 2 \mathbb{Z}, \mathbb{Z} / 3 \mathbb{Z}$ or $n \geq 2 ; D_{i} \cong \mathbb{Z} / 2 \mathbb{Z}$ or $\mathbb{Z} / 3 \mathbb{Z}$. If $D_{i} \not \mathbb{Z} / 2 \mathbb{Z}, \mathbb{Z} / 3 \mathbb{Z}$, it follows from Lemma 2 that for any $\gamma \in M_{n_{i}}\left(D_{i}\right)$, there exists $\alpha \in G L_{n_{i}}\left(D_{i}\right)$ such that $\gamma+\alpha, \gamma-\alpha^{-1} \in G L_{n_{i}}\left(D_{i}\right)$. If $n \geq 2 ; D_{i} \cong \mathbb{Z} / 2 \mathbb{Z}$ or $\mathbb{Z} / 3 \mathbb{Z}$, it follows from the preceding discussion and Example 8 that for any $\gamma \in M_{n_{i}}\left(D_{i}\right)$, there exists $\alpha \in G L_{n_{i}}\left(D_{i}\right)$ such that $\gamma+\alpha, \gamma-\alpha^{-1} \in G L_{n_{i}}\left(D_{i}\right)$. Thus, for any $\gamma \in R$, there exists $\bar{\alpha} \in U(R / J(R))$ such that $\bar{\gamma}+\bar{\alpha}, \bar{\gamma}-\bar{\alpha}^{-1} \in U(R / J(R))$. As units lift modulo $J(R)$, we conclude that there exists $\alpha \in U(R)$ such that $\gamma+\alpha, \gamma-\alpha^{-1} \in U(R)$, as needed.

COROllary 10. Let $A$ be an artinian right $R$-module. If $\frac{1}{2}, \frac{1}{3} \in R$, then for any $\gamma \in \operatorname{End}_{R}(A)$, there exists $\alpha \in \operatorname{Aut}_{R}(A)$ such that $\gamma+\alpha, \gamma-\alpha^{-1} \in A u t_{R}(A)$.

Proof. Construct two $R$-morphisms $\varphi: A \rightarrow A$ given by $\varphi(a)=a \cdot \frac{1}{2}$ and $\phi: A \rightarrow$ $A$ given by $\phi(a)=a \cdot \frac{1}{3}$ for any $a \in A$. It is easy to verify that $\varphi, \phi \in A u t_{R}(A)$, i.e. $\frac{1}{2}, \frac{1}{3} \in \operatorname{End}_{R}(A)$. As is well known, $\operatorname{End}_{R}(A)$ is semi-local. Therefore, we complete the proof by Proposition 9.

For a semi-local ring $R$, it follows from Proposition 9 that $R$ satisfies GoodearlMenal condition if and only if for any $\gamma \in R$, there exists $\alpha \in U(R)$ such that $\gamma+\alpha, \gamma-$ $\alpha^{-1} \in U(R)$. This should be contracted to the following simple fact: a semi-local ring $R$ satisfies unit 1-stable range if and only if for any $\gamma \in R$, there exists $\alpha \in U(R)$ such that $\gamma-\alpha, \gamma-\alpha^{-1} \in U(R)$. Let $V$ be a countably generated right vector space over a division ring $D$, and let $\gamma \in \operatorname{End}_{D}(V)$. The preceding observation also raises a problem: if $D \neq \mathbb{Z} / 2 \mathbb{Z}$, is there some $\alpha \in A u t_{D}(V)$ such that $\gamma-\alpha, \gamma-\alpha^{-1} \in A u t_{D}(V)$ ?

\section{REFERENCES}

1. H. Chen, On exchange rings with all idempotents central, Algebra Colloq. 6 (1999), $45-50$. 
2. H. Chen, Units, idempotents, and stable range condition, Comm. Algebra 29 (2001), 703-717.

3. K. R. Goodearl, Von Neumann Regular Rings, Pitman, London-San FranciscoMelbourne, 1979; 2nd ed., Krieger, Malabar, FL, 1991.

4. J. Han and W. K. Nicholson, Extensions of clean rings, Comm. Algebra 29 (2001), $2589-2595$.

5. W. K. Nicholson and K. Varadarjan, Countable linear transformations are clean, Proc. Amer. Math. Soc. 126 (1998), 61-64.

6. D. Zelinsky, Every linear transformation is a sum of nonsingular ones, Proc. Amer. Math. Soc. 5 (1954), 627-630. 